\title{
POSIBILIDADES DE USO DE LA RED SOCIAL DISCIPLINAR: PERSPECTIVAS DE DOCENTES
}

\author{
José Manuel Meza Cano y Enimia Virgen López Cruz \\ Universidad Nacional Autónoma de México \\ México
}

\begin{abstract}
RESUMEN
Se analizan las posibilidades de uso de una Red Social Disciplinar desde la perspectiva de cuatro docentes de módulos propedéuticos, para ello se elaboró una entrevista de siete reactivos abiertos para profesores de la carrera en Psicología en Línea de la UNAM. Para el marco teórico se revisaron los postulados de la Teoría de la Actividad Histórico-cultural (CHAT), el uso de las redes sociales en el ámbito educativo y la definición de Red Social Disciplinar. Los ejes de la entrevista corresponden a: medios de comunicación profesor-alumno, beneficios de la Red Social Disciplinar, características y posible uso dentro de la programación de actividades. Se realizaron tres fases de análisis: una descriptiva sobre las respuestas de la entrevista, una empleando análisis del discurso y una de análisis con respecto al marco de la CHAT. Se obtuvo que tres los profesores consideran que una Red Social Disciplinar puede tener beneficios para la comunicación entre profesor-estudiante y entre pares. Se obtuvieron 89 segmentos categorizados, siendo los más frecuentes las dificultades para realizar las prácticas del módulo seguido de los beneficios del uso de una red social disciplinar, a partir de estos datos se caracterizaron las posibilidades de uso de las redes sociales disciplinares con respecto al marco de la CHAT. Se concluye que son los docentes quienes deben plantear el objetivo y propósito académico específico de cada asignatura en la que se emplearía la red, así como la necesidad de crear este tipo de espacios virtuales seguros para la interacción profesor-alumno.
\end{abstract}

Palabras Clave: Redes sociales, educación a distancia, profesores, Internet

\section{POSSIBILITIES OF USING THE DISCIPLINARY SOCIAL NETWORK: TEACHERS' PERSPECTIVES}

\begin{abstract}
The possibilities of using a Disciplinary Social Network are analyzed from the perspective of four teachers of preparatory modules, for which an interview of seven open questions was prepared for teachers of the Online Psychology degree at UNAM. For the theoretical framework, the postulates of the Historical-Cultural Activity Theory (CHAT), the use of social networks in the educational field and the definition of the Disciplinary Social Network were reviewed. The axes of the interview correspond to: teacher-student communication media, benefits of the Disciplinary Social Network, characteristics and possible use within the programming of activities. Three phases of analysis were carried out: a descriptive one with the interview responses, one using discourse analysis and one of analysis with respect to the CHAT framework. It was obtained that three teachers consider that a Disciplinary Social Network can have benefits for communication between teacher-student and among peers. 89 categorized segments were obtained, the most frequent being the difficulties to carry out the module practices followed by the benefits of the use of a disciplinary social network, from these data the possibilities of using disciplinary social networks were characterized with respect to the CHAT framework. It is concluded that it is the teachers who must raise the objective and specific academic purpose of each subject in which the network would be used, as well as the need to create this type of safe virtual spaces for teacher-student interaction. Keywords: Disciplinary Social Network, distance education, teachers, Internet
\end{abstract}

Bitácora del Artículo:

| Recibido: 4 de Agosto de 2021 | Aceptado: 9 de Noviembre de 2021 | Publicado en línea: Enero - Junio de 2022 | 


\title{
Autoría y Derechos de Propiedad Intelectual
}

\section{CONOCIMIENTOS Y PERCEPCIÓN DE ESTUDIANTES DE PSICOLOGIIA SOBRE EL TRABAJO PROFESIONAL EN PERSONAS CON VIH}

\author{
Ricardo Sánchez Medina, María de los Ángeles Estrada Martínez, Consuelo Rubi Rosales Piña, \\ David Javier Enríquez Negrete y Maria Fernanda Lozano Quiroz \\ Universidad Nacional Autónoma de México \\ México
}

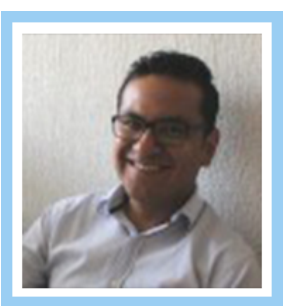

José Manuel Meza Cano FES- Iztacala, UNAM

Correo: manuel.meza@iztacala.unam.mx

Doctor en Psicología por la Facultad de Psicología, UNAM, área de Psicología Educativa y del Desarrollo. Licenciado en psicología por la Facultad de Estudios Superiores Iztacala, UNAM y Profesor Titular "A" Tiempo Completo en la misma dependencia. Miembro del Sistema Nacional de Investigadores, Nivel 1. Sus líneas de trabajo se enmarcan en Entornos Personales de Aprendizaje, aprendizaje autorregulado, creencias epistemológicas, herramientas de internet para el aprendizaje, educación en línea y a distancia, diseño instruccional, redes sociodigitales aplicadas a la educación y Teoría de la Actividad Histórico-Cultural en medios sociodigitales.

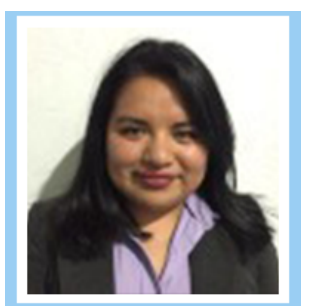

Enimia Virgen López Cruz

FES- Iztacala, UNAM

Correo: enylopez123@gmail.com
Licenciada en psicología egresada de la Facultad de estudios superiores Iztacala UNAM del sistema SUAYED. Cursó los campos formativos en psicología educativa y psicología organizacional. Participó en dos estancias de investigación en la fundación FONABEC centro CODAF Magdalena Contreras con estudiantes de bachillerato a distancia UNAM sobre el tema habilidades sociales y el segundo tema en comprensión lectora y búsqueda de información en Internet. Participó con una ponencia en el XXVIII Encuentro Internacional de Educación a Distancia por la Universidad de Guadalajara sobre el tema de redes sociales y su implementación en la educación a distancia para la enseñanza en psicología.

\section{CONTRIBUCIÓN DE lOS AUtORES}

José Manuel Meza Cano Planteamiento general de la investigación, desarrollo del marco teórico, análisis de resultados y desarrollo de las conclusiones. Enimia Virgen López Cruz Desarrollo del marco teórico, contacto con la muestra, levantamiento de entrevistas, análisis de resultados.

\section{AGRADECIMIENTOS}

Trabajo realizado gracias al Programa UNAM-PAPIME <PE305121> "Entornos y Redes Personales de Aprendizaje (ERPA) aplicados a proyectos de titulación".

\section{Datos de Filiación de los Autores}

Facultad de Estudios Superiores Iztacala, UNAM

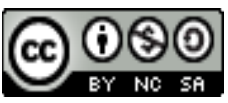

Copyright: (c) 2022 Meza - Cano, J. M., Lopez-Cruz, E. V..

Este es un artículo de acceso abierto distribuido bajo los términos de la licencia Creative Commons Reconocimiento-NoComercial 4.0 Internacional, por lo que su contenido gráfico y escrito se puede compartir, copiar y redistribuir total o parcialmente sin necesidad de permiso expreso de sus autoras con la única condición de que no se puede usar con fines directamente comerciales y los términos legales de cualquier trabajo derivado deben ser los mismos que se expresan en la presente declaración. La única condición es que se cite la fuente con referencia a la Revista Digital Internacional de Psicología y Ciencia Social y a sus autoras. 


\section{TABLA DE CONTENIDO}

Redes sociales en el ámbito educativo y redes sociales disciplinares , 4

Teoría de la Actividad Histórico Cultural , 5

MÉTODO

Participantes, 7

Procedimiento, 7

Análisis de resultados, 7

RESULTADOS

Resultados de la fase 1. Descripción de las respuestas por

docente., 8

Resultados de la fase 2. Frecuencia y ejemplos de segmentos

categorizados., 8

Resultados de la fase 3. Análisis de acuerdo con la CHAT., 8

Discusión

CONCLUSIONES

REFERENCIAS 


\section{Redes sociales en el ámbito educativo y redes sociales disciplinares}

n este artículo se presentan los resultados de entrevistas que abordan un análisis para el uso de una Red Social Disciplinar enfocada a estudiantes universitarios en educación en línea desde la perspectiva de los profesores, con la finalidad de identificar elementos que pueden favorecer el uso de esta red como un elemento didáctico a ser tomado en cuenta en el futuro, especialmente en módulos propedéuticos, que corresponden al cuarto semestre de la licenciatura en psicología en línea. Esto es relevante dado que estos módulos son el primer acercamiento al campo aplicado de la psicología en sus diferentes áreas como son clínica, educativa, organizacional, NEE y salud. El marco teórico desde el cual se propone el análisis de los datos es la Teoría de la Actividad Histórico Cultural.

Dentro de la carrera licenciatura en línea de SUAyED se parte de una Plataforma Única de Conocimientos Básicos (PUCB), la cual conforma los primeros cuatro semestres, en donde se cursan módulos principalmente teóricos, para luego dar paso a los campos de profundización en diferentes áreas de la psicología como son: educativa, salud, necesidades educativas especiales, clínica y organizacional. Estos campos de profundización contemplan los semestres quinto a noveno (Universidad Nacional Autónoma de México, 2005). Dentro de la PUCB, en el cuarto semestre, se ubican los módulos propedéuticos, los cuales tienen objetivos generales comunes relacionados con que los estudiantes obtengan conocimientos teóricos, metodológicos y aplicados previos a su ingreso en las áreas profundización, a través de esto se busca que obtengan un panorama general para tomar la decisión sobre el área de énfasis en psicología para su formación profesional. Durante estos módulos los estudiantes deben realizar una estancia en escenarios propios de las áreas de profundización, principalmente como asistente, por lo que esta es la primera vez que se enfrentan a áreas de servicio o investigación en las áreas de su interés.

Dada la importancia de ser el primer acercamiento de los estudiantes al campo aplicado, se tuvo el interés de proponer un espacio donde puedan consultar, compartir y construir conocimientos que puedan aplicar en su primera estancia de investigación y estancia de servicio, al mismo tiempo que pueden comunicarse con docentes de su módulo u otros módulos, aspecto que no puede realizarse en una plataforma Moodle. Por esta razón se propone una red social disciplinar. A continuación se profundizará en este término.
Las redes sociales que emplean tecnologías de información y comunicación (TIC) en su configuración se definen, según autores como (Peña, Pérez y Rondón, 2010), como una estructura social que emplea recursos de Internet, favoreciendo las relaciones entre individuos o grupos que comparten objetivos e intereses similares, ya sea conectando con personas que se han conocido fuera de la web o como medio para conectar con nuevas personas.

Dada su popularidad y alcance, se encuentran diversos debates y experiencias de implementación de las redes sociales para el ámbito educativo, por ejemplo, en el artículo de Ormart y Navés (2014) dan nuevas discusiones con el análisis de las ventajas que dan las redes sociales a la educación superior pues genera una modificación en el modo de la interrelación, promueve compartir información relevante, promueve el sentido crítico e incentiva la investigación como actividad formativa. En este estudio la red social libre que usaron fue la plataforma NING en donde crearon diferentes grupos para alumnos de la licenciatura en psicología, para docentes y para colaboradores, en donde su trabajo fue distribuido a través de texto, muro de comentarios y foros de discusión. Como conclusión los autores consideran que la inclusión de las redes sociales en los modelos de formación cumple dos funciones principales, la primera es que favorece las competencias relacionadas con contextos sociales y laborales, mientras que la segunda función enfatiza en la actualización situada y contextual sobre el desarrollo contemporáneo de la psicología.

Otro ejemplo en donde se aborda el uso de las redes sociales como escenario para favorecer aprendizajes significativos en entornos digitales de aprendizaje es el trabajo de Oliva (2017), quien destaca que las redes sociales permiten el aprendizaje colaborativo, se realizó un análisis de las redes sociales digitales más empleadas en El Salvador, siendo Facebook el más empleado, sin embargo el autor también señala los riesgos en su uso, por ejemplo, dispersión y asumir información no confiable, administración y gestión de cuentas y por último la adaptación a la red social, aunque como ventajas afirma que son un ambiente educativo que promueven la dinámica de trabajo al acercar el aprendizaje no-formal y el formal, en donde el estudiante puede atender las exigencias de su propia educación. El autor concluye que es necesario el desarrollo de Entornos Personales de Aprendizaje en redes sociales y, por otro lado, se debe favorecer la socialización de información y generación de conocimientos. 
En otro trabajo de Marín y Cabero (2019) se presenta un análisis sobre la visión negativa que pueden tener alumnos y docentes respecto a la seguridad del usuario, privacidad e información que se comparte en redes sociales para el ámbito educativo. Para atender estos aspectos los autores enfatizan en que se requieren más estudios y análisis desde dos perspectivas: como elemento de innovación en el acto educativo enfocándose a las metodologías del proceso enseñanza-aprendizaje y, por otra parte, la presentación de estudios sobre los resultados de investigaciones sobre el uso de las redes sociales con énfasis académico.

En esta misma línea y hablando de los posibles riesgos, a decir de Muñoz (2018), las grandes corporaciones actuales (Google, Facebook, Microsoft, Amazon y Apple, sobre todo), dueñas de las principales redes sociales, acumulan gran cantidad de datos obtenidos a partir del comportamiento de los usuarios, los procesan y obtienen de ellos información para prestar otros servicios altamente rentables. Estas empresas buscan el control de plataformas, servicios e infraestructura mundial, ejemplo de ello es Facebook, quien mantiene un modelo de negocio basado en los datos de los millones de usuarios que tienen cuentas en esta red, para luego, ser cedidos a terceros.

Bajo este panorama, autores como Cabrera (2017), señalan la urgencia de visibilizar y dar soporte a las plataformas descentralizadas desde las instituciones educativas públicas, en donde las universidades que cuentan con infraestructura suficiente, puedan generar sus propios sistemas de comunicación, migrando a modelos autogestionados a partir de redes de código abierto, buscando la autonomía tecnológica y la neutralidad de la red. A decir de este autor, las redes privativas como Facebook no sólo mercantilizan los datos de los usuarios, sino que impiden el desarrollo de actividades de investigación, dado que no es posible analizar los datos generados por los participantes, pues no es posible ingresar a las bases de datos, sólo a través de servicios de terceros, que también son de pago.

En este sentido, se creó el concepto de Red Social Disciplinar, la cual se caracteriza por dos postulados principales: una comunidad en donde se discute sobre un área de conocimiento en específico y el software libre que lo permite, abriendo así las posibilidades de autogestión, autocontrol y autogobernanza de la red, es decir, es la comunidad de usuarios quienes pueden definir los términos de uso, los aspectos de censura y moderar el acceso de acuerdo con parámetros definidos por ellos mismos. Según Meza, Miranda y Delgado (2019) la intención de una Red Social Disciplinar es que los usua- rios puedan intercambiar información, discutir y llegar a consensos y hasta generar conocimiento sobre temas específicos, en este caso, la discusión de temas relacionados con psicología. Esto puede ser analizado a partir de marcos teóricos y metodológicos como es el caso de la Teoría de la Actividad Histórico Cultural, la cual proporciona una metodología para la investigación e intervención, tomando en cuenta elementos como: el sujeto, las reglas, la comunidad y la división de trabajo.

\section{Teoría de la Actividad Histórico Cultural}

A grandes rasgos, se desarrollaron tres generaciones de la Teoría de la Actividad Histórico Cultural (CHAT). La primera corresponde al modelo propuesto por $\mathrm{Vy}$ gotsky (citado en Miranda, 2015), en el cual representa la relación sujeto-objeto para el logro de un objetivo a través de herramientas y artefactos mediadores, posteriormente, en la segunda generación lo característico es que Leontiev hizo la distinción entre acción, actividad y operación, mientras que la actividad de los individuos está impulsada por objetivos o motivos. Según Miranda (2015) Leontiev continua con el desarrollo señalando la necesidad de una unidad de análisis a partir de la interacción de tres elementos: actividad (es decir el motivo), acción (el objetivo que se busca) y operación (incluyendo las condiciones en donde se lleva a cabo la acción). Por último, en la tercera generación, es Engeström el que pone el foco en herramientas dialógicas que dan paso a la diversidad de voces y perspectivas, así como puntos de vista entre los sistemas relacionados en interacción (Erausquin, 2014).

En Larripa y Erausquin (2008), se afirma que los principales principios la teoría de la actividad, en su última generación, son que considera al sistema de actividad como la unidad mínima de análisis psicológico, en donde es fundamental la división de trabajo para la multiplicidad de voces, dando paso al cambio, considerando la historia local de la actividad, del objeto e instrumentos, son necesarias las fuentes de cambio y desarrollo para las acciones de innovación en el sistema, dando paso al aprendizaje expansivo, siendo adaptaciones dentro de los sistemas.

Es así que Engeström acuña el término "sistema de actividad" para referirse a la distribución e intercambio dentro de una comunidad a partir de los elementos como las reglas internas, la ejecución de roles que dan paso a la división del trabajo, encaminados al logro del objetivo y su posible transformación (Salas, 2016). En palabras de Miranda (2015), el sistema que replantea Engeström a partir de los aportes de Leontiev se puede representar de la siguiente manera en un triángulo, en 
donde todos los elementos interactúan entre sí (figura 1): se tiene al sujeto en una situación de aprendizaje, los artefactos mediadores o instrumentos que se usa durante la actividad, el objeto representado por contenidos y que son los que dirigen la actividad, la comunidad a la que pertenece este sujeto, las reglas determinadas por la comunidad y los roles que delimitan la división del trabajo para lograr las tareas.

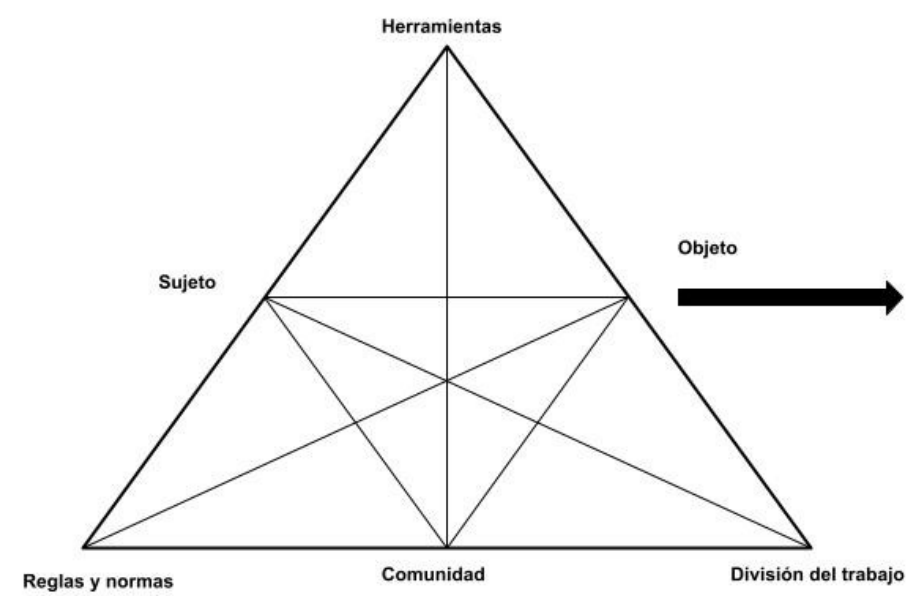

\section{Figura 1.}

Modelo de la tercera generación de la Teoría de la Actividad Histórico Cultural (obtenida de Engeström, 2009).

Salas (2016) afirma que los elementos de la CHAT permiten analizar el desarrollo de la actividad humana en su contexto, puesto que los humanos utilizan procedimientos que encadenan los medios, herramientas e instrumentos para el logro del objetivo, tomando en cuenta las condiciones del grupo, pero también de los individuos. Por lo tanto, bajo esta teoría los artefactos mediadores van desde instrumentos materiales hasta simbólicos, a disposición de los sujetos para realizar el trabajo sobre el objeto. Todo esto regido bajo condiciones ambientales y sociales en donde la actividad es desarrollada. A lo interno del sujeto se pueden mencionar las motivaciones y reacciones psico-afectivas, dando como producto del sistema el resultado de estos elementos interactuantes.

La CHAT permite conocer a los sujetos, su ubicación y las razones de su esfuerzo en un contexto social, los resultados de su aprendizaje, las formas o caminos a partir de los cuales logran este aprendizaje y el impacto que esto conlleva (Engeström, 2001).

Algunos autores han empleado la Teoría de la Actividad para explorar la relación entre las prácticas humanas y el ámbito tecnológico. Por ejemplo, García (2017) en su estudio nombrado aplicabilidad de la CHAT en los estudios de comportamiento informacional concluye que esta teoría aún ha sido poco explorada pero es útil para conocer a los sujetos en su contexto, dadas las posibilidades de análisis que presenta. Empleó esta teoría como una herramienta tanto teórica como metodológica para analizar y dar cuenta de las formas en que los individuos realizan búsquedas, almacenan, recuperan y emplean información de su interés. En su estudio empleó dos conceptos, el de comportamiento informacional y por otro lado la actividad humana, de tal modo que afirma que la actividad humana es también un comportamiento de tipo informacional.

En otro estudio, Cunha Jr., Van Oers y Kontopodis (2016) emplearon la CHAT para analizar cómo Facebook puede emplearse para establecer nuevas formas de comunicación entre alumnos y profesores universitarios. Entre sus principales hallazgos enfatizan la creación de normas y reglas claras para todos los participantes, especialmente al realizar actividades académicas mediadas por esta plataforma social. Estas normas deben ser establecidas por los docentes dado que favorece la creación de la comunidad, delimitando las acciones y roles, Ilevando la actividad académica a un proceso de logro de cara a un objetivo cultural común.

Partiendo de este escenario, este estudio pretende abordar un área de oportunidad para que estudiantes y profesores universitarios conozcan los alcances de las redes sociales disciplinares como espacios colaborativos de aprendizaje. Además de las posibilidades que brindan estos espacios, también sería favorable para las instituciones, dado que se señala la falta de uso de redes sociales de código abierto en las universidades, tanto públicas como privadas. Como revisó anteriormente, los estudiantes consultan en su mayoría Facebook pese a que no cumple cabalmente con las características deseables para ser usado como red social con énfasis académico como es la venta de información a terceros o la presentación de información diferenciada dado el algoritmo propio de estas redes (Meza et al., 2015; Kirschner, 2015).

Sin embargo, si de integrar las redes sociales en el acto educativo se trata, autores como Vázquez y Cabero (2015) y Suárez, Carreño y Rivero (2017), enfatizan que es el docente quien tiene que plantearse el objetivo didáctico para guiar el uso, fungiendo como facilitador, conociendo previamente las posibilidades de las redes a nivel tecnológico y contar con las habilidades necesarias para guiar a los participantes en favor de su aprendizaje.

Por lo tanto, el objetivo de este trabajo es analizar las posibilidades de uso de las redes sociales disciplinares desde la perspectiva de los docentes que imparten módulos propedéuticos. 


\section{MÉtodo}

Se trata de un estudio con diseño no experimental, con un alcance exploratorio.

\section{Participantes}

Participaron 4 profesores de la licenciatura en psicología en la modalidad en línea de SUAyED. Por lo tanto, se trata de una muestra no-probabilística, de sujetos voluntarios, ya que se envió un correo electrónico a los profesores que impartían módulos propedéuticos y se recibió respuesta de los cuatro profesores.

A continuación se describe de manera general el perfil de los cuatro profesores participantes.

1. Profesor 1. Licenciada en psicología por la BUAP con Maestría en Psicología con residencia en terapia familiar en la UNAM.

2. Profesor 2. Licenciada en psicología con Maestría en Psiclogía en el área de Educación Especial.

3. Profesor 3. Licenciado en psicología y Maestría en Ciencias.

4. Profesor 4. Licenciada en psicología con formación en psicología clínica con especialidad en psicoterapia familiar con enfoque sistémico además de un entrenamiento en terapia narrativa.

\section{Materiales}

Consta de una entrevista semi-estructurada con siete reactivos de preguntas abiertas referentes a áreas correspondientes a plataforma Moodle, la cual es el medio de comunicación con estudiantes usual, beneficios posibles del uso redes sociales disciplinares, posibilidades de participación de profesores y alumnos en la red social disciplinar y, por último, aspectos del programa de actividades de los módulos propedéuticos que pueden beneficiarse de una red social disciplinar. Se convocó al tutor de manera individual y se realizó cada entrevista a través de videoconferencia. Cada entrevista fue grabada en audio con un teléfono celular, y se le informó al profesor sobre la confidencialidad de los datos del estudio; en promedio las entrevistas tuvieron una duración de 40 minutos. Las preguntas realizadas se muestran a continuación:

1. Adicional a la plataforma, ¿Qué otro medio de comunicación utiliza para estar en contacto con los estudiantes?

2. En su experiencia ¿Cuáles son las dificultades que presentan los estudiantes al estudiar a distancia?

3. Cree que los estudiantes podrían obtener algún beneficio al emplear redes sociales como apoyo para la interacción entre ellos? Si su respuesta es afirmativa ¿Cuál o cuáles?
4. Qué características considera que debe tener la Red Social Disciplinar para apoyar al aprendizaje y la interacción de los estudiantes?

5. Consideraría participar en la Red Social Disciplinar como gestor de conocimientos en su rol como docente?

6. Qué tipo de tipo de actividad sería pertinente implementar en la programación de actividades empleando la red social disciplinar?

7. Como profesor de la licenciatura en psicología en línea ¿Cuál considera que sería el apoyo brindado para los estudiantes en la Red Social Disciplinar en relación con compartir conocimientos y experiencias?

\section{Procedimiento}

Se contactó a través de correo electrónico a los profesores de los módulos propedéuticos que se encuentran activos actualmente en la plantilla académica de la carrera de psicología de SUAyED, FES Iztacala, en este primer correo se expuso brevemente el objetivo y naturaleza de la entrevista a realizar. Para aquellos docentes que respondieron al primer correo se les solicitó un horario en el cual se realizó la entrevista a través de Google Meet, la cual fue grabada en audio, previo consentimiento del profesor.

\section{Análisis de resultados}

El análisis se centró en tres fases. En la primera las entrevistas fueron transcritas textualmente, a partir de la versión estenográfica se realizó el análisis por dos jueces quienes crearon categorías generales para agrupar las respuestas de los cuatro profesores de acuerdo con cada reactivo de la entrevista realizada. Posteriormente, ambos jueces se reunieron para ajustar las categorías y los resultados agrupados en cada una. Para un análisis más exhaustivo, se realizó la segunda fase, en donde se procedió al análisis de discurso dentro de cada entrevista, tomando como unidad mínima la oración, empleando para ello la plataforma Taguette (https://taguette.org) la cual permite seleccionar segmentos de texto y asignarlo a categorías. Con ello ambos jueces realizaron el análisis de una entrevista por separado, se reunieron una primera vez, ajustaron las categorías discutiéndolas, para luego realizar el análisis de las tres entrevistas restantes por separado. Para la tercera fase, se enmarcaron los resultados de las fases anteriores de acuerdo con los elementos de la CHAT, para visualizar así las posibilidades de uso de la red social disciplinar como un Sistema de Actividad. 


\section{Resultados}

Se generaron 6460 palabras a partir de la transcripción de las cuatro entrevistas.

Resultados de la fase 1. Descripción de las respuestas por docente.

Posterior al agrupamiento de las respuestas obtenidas de las entrevistas en categorías generales, los cuales son presentados posteriormente, de manera grupal, empleando para ello una tabla.

\section{Medios de comunicación}

Tres de los cuatro profesores mencionaron no hacer uso de las redes sociales, únicamente usan el mensajero de la plataforma y correos para comunicarse con los estudiantes. Algunos otros medios que suelen usar son Google Hangouts, aulas virtuales CUAED y WhatsApp. Un profesor mencionó usar Facebook.

Dificultades identificadas para aprobar los módulos propedéuticos

Las problemáticas son muy diversas algunas de las que se mencionaron son: el acceso al escenario de práctica profesional, la zona geográfica donde viven los estudiantes y falta de conocimientos metodológicos.

Beneficios que obtendrán los estudiantes al participar en la red social disciplinar

Los cuatro profesores mencionaron que la red social disciplinar sí puede tener beneficios para el estudiante ya que permitiría trabajar de forma colaborativa para aclarar sus dudas, compartir su experiencia y compartir material bibliográfico.

\section{Características de la red social disciplinar}

Los cuatro profesores resaltaron que sería importante depurar los usuarios en la red al finalizar cada semestre, es decir, remover a los que concluyeron el módulo. También mencionaron que sería de vital importancia compartir las normas y reglas de participación con los estudiantes y se confirme su revisión ya que de esa forma se logrará que el estudiante comprenda cómo debe participar.

\section{Ajustes en la programación de actividades}

Los profesores mencionaron que es posible hacer cambios en la programación de actividades de su módulo para diseñar actividades con la el objetivo de favorecer la participación activa de los estudiantes en la red social disciplinar.

\section{Participación en la red social disciplinar con un rol de docente}

Se encontró que los cuatro profesores están dispuestos a participar en la red social disciplinar no sólo como gestor de conocimientos sino también como un participante más, con el objetivo de identificar las dudas de los estudiantes.

\section{Temas relevantes a tratar en la red social disciplinar}

De acuerdo con los cuatro profesores los temas a revisar en la red social disciplinar van enfocados a los aspectos teóricos, metodológicos y aplicados de cada área de la psicología.

Periodo para habilitar la red social disciplinar

Sólo tres de los cuatro profesores consideran que lo ideal es habilitar la red social disciplinar al inicio de semestre ya que consideran que está puede dar una introducción a la asignatura, además de que creará expectativas de la participación del estudiante en las estancias de investigación y servicio. El profesor restante mencionó que es pertinente que se habilite la red social disciplinar durante la práctica, ya que de esta forma el estudiante participará en la red social disciplinar con dudas sobre su estancia. Además los profesores consideran que la red social disciplinar puede estar habilitada por un corto tiempo para evitar saturación de trabajo a estudiantes.

A continuación, en la tabla 1, se muestran en forma de resumen los aspectos relevantes de los resultados de las entrevistas:

\section{Resultados de la fase 2. Frecuencia y ejemplos de segmentos categorizados.}

De acuerdo con lo obtenido a partir del análisis del discurso empleando Taguette, se obtuvieron un total de 89 segmentos categorizados. Se aprecia que la categoría con mayor frecuencia de segmentos categorizados fue "Dificultades para la práctica" con 21 segmentos, en la que los docentes mencionan diversos obstáculos que los estudiantes de los módulos propedéuticos enfrentan, lo cual da pie a conocer las problemáticas cotidianas, le siguió la categoría de "Beneficios de la red social disciplinar" con 15 segmentos, en donde los docentes mencionaron las ventajas y utilidades que una red social disciplinar podría tener para los alumnos en el marco de los módulos propedéuticos que imparten. Cabe señalar que solamente un docente mencionó ventajas de las redes sociales comunes, lo cual tuvo un único segmento categorizado. Los segmentos categorizados y un ejemplo de ellos fueron agrupados en la tabla 2.

\section{Resultados de la fase 3. Análisis de acuerdo con la CHAT.}

Una vez descritos los principales resultados, es importante caracterizarlos desde el marco teórico de la CHAT, para así visualizar la propuesta de la Red Social Disciplinar como un sistema de actividad. 
Tabla 1.

Categorías relevantes en las entrevistas realizadas.

\begin{tabular}{|c|c|c|c|c|}
\hline Categoría & Profesor 1 & PrOFESOR 2 & PrOFESOR 3 & PrOFESOR 4 \\
\hline Medios de comunicación & $\begin{array}{l}\text { Mensajero de } \\
\text { plataforma. } \\
\text { Foro } \\
\text { Correo }\end{array}$ & $\begin{array}{l}\text { Mensajero de } \\
\text { plataforma. } \\
\text { Hangouts } \\
\text { Correo electrónico } \\
\text { Llamada } \\
\text { WhatsApp }\end{array}$ & $\begin{array}{l}\text { Mensajero de } \\
\text { plataforma. } \\
\text { Facebook } \\
\text { WhatsApp }\end{array}$ & $\begin{array}{l}\text { Mensajero de } \\
\text { plataforma } \\
\text { Aulas virtuales de } \\
\text { CUAED }\end{array}$ \\
\hline $\begin{array}{l}\text { Dificultades al estudiar a } \\
\text { distancia }\end{array}$ & $\begin{array}{l}\text { Limitaciones en la } \\
\text { práctica. } \\
\text { Falta de } \\
\text { conocimientos } \\
\text { metodológicos }\end{array}$ & $\begin{array}{l}\text { Limitaciones en la } \\
\text { práctica. } \\
\text { Falta de conocimientos } \\
\text { metodológicos. }\end{array}$ & $\begin{array}{l}\text { Zona geográfica donde } \\
\text { vive el estudiante. } \\
\text { Limitaciones en la } \\
\text { práctica. }\end{array}$ & $\begin{array}{l}\text { Limitaciones en la } \\
\text { práctica. }\end{array}$ \\
\hline $\begin{array}{l}\text { Beneficios que } \\
\text { obtendrán los } \\
\text { estudiantes }\end{array}$ & Compartir experiencia. & $\begin{array}{l}\text { Colaboración para aclarar } \\
\text { dudas }\end{array}$ & $\begin{array}{l}\text { Colaboración para aclarar } \\
\text { dudas }\end{array}$ & $\begin{array}{l}\text { Espacio fuera de } \\
\text { plataforma para aclarar } \\
\text { dudas }\end{array}$ \\
\hline $\begin{array}{l}\text { Características de Red } \\
\text { Social Disciplinar }\end{array}$ & $\begin{array}{l}\text { Lineamientos (Reglas } \\
\text { y normas) }\end{array}$ & $\begin{array}{l}\text { Lineamientos (Reglas y } \\
\text { normas) } \\
\text { Retroalimentación por } \\
\text { experto o tutor. }\end{array}$ & $\begin{array}{l}\text { Lineamientos (Reglas y } \\
\text { normas) } \\
\text { Responsabilidad para } \\
\text { compartir información } \\
\text { Depuración de } \\
\text { participantes }\end{array}$ & $\begin{array}{l}\text { Lineamientos (Reglas y } \\
\text { normas) }\end{array}$ \\
\hline $\begin{array}{l}\text { Programación de } \\
\text { actividades }\end{array}$ & Iniciar discusión & Compartir recursos & $\begin{array}{l}\text { Practicar con } \\
\text { compañeros }\end{array}$ & Uso como un foro \\
\hline $\begin{array}{l}\text { Participar en la Red } \\
\text { Social Disciplinar con rol } \\
\text { de docente }\end{array}$ & Sí & Sí & Sí & Sí \\
\hline Temas relevantes & Entrevista & $\begin{array}{l}\text { Cómo realizar cartas } \\
\text { descriptivas. } \\
\text { Tipos de observación }\end{array}$ & Ejercicios de entrevista & Establecer formatos \\
\hline $\begin{array}{l}\text { Periodo para aplicar la } \\
\text { red social disciplinar }\end{array}$ & Durante el semestre & Al principio del semestre & Al principio del semestre & $\begin{array}{l}\text { Al principio del } \\
\text { semestre y sólo por } \\
\text { corto tiempo. }\end{array}$ \\
\hline
\end{tabular}

Desde el marco de la Teoría de la Actividad se puede realizar un análisis del uso propuesto por los profesores de la red social disciplinar, de acuerdo con cada uno de sus elementos (ver figura 2).

Como puede verse en la figura 2, estos elementos han sido obtenidos a partir de las entrevistas realizadas con los profesores. A decir de ellos, cada elemento sería constituido de la siguiente manera:

Sujeto: está conformado por los estudiantes y profesores de las asignaturas, dado que son las figuras que buscan el objeto común.

Objeto: Compartir y discutir información relevante de acuerdo al temario de la asignatura. Este objeto se encuentra a su vez ligado a un resultado, que construir conocimiento.
Resultado: Construcción de conocimientos. Específicamente, al ser una Red Social Disciplinar, estos conocimientos son propios del área a la cual se enfoca el sistema, en este caso conocimientos tanto teóricos como metodológicos sobre prácticas en psicología.

Artefactos mediadores: En este caso el principal artefacto es la Red Social Disciplinar. Al hablar de su implementación se puede recurrir a redes sociales libres y de código abierto, como por ejemplo Red social libre Mastodon, la cual puede ser descargada e instalada en un servidor local o en la nube (https://joinmastodon.org/).

Reglas y normas: Se definen por los lineamientos para la participación y los términos de uso de la red, los cuales tendrán que ser desarrollados por los administradores de la red, quienes a su vez pueden ser los 
Tabla 2.

Análisis sobre las entrevistas empleando análisis del discurso, frecuencias y ejemplos de las categorías con base en la respuesta de los profesores.

CATEgoría FrECUENCIA EJEMPLO

$\begin{array}{ll}\begin{array}{l}\text { Dificultades de la } \\ \text { práctica }\end{array} & 21 \\ & \begin{array}{l}\text { "La parte de encontrar un lugar o en este caso a un psicólogo que le permite hacer estas } \\ \text { observación, pero gran parte del grupo se queda solamente en la entrevista" }\end{array}\end{array}$

Beneficios de la red $15 \quad$ "Compartir la experiencia eso me parece genial porque aunque ya es parte de algo que social disciplinar nosotros hacemos en foros pero si en esta red social es como que ellos se van a sentir su espacio sin estar siendo evaluados creo que les puede dar mucha libertad para acudir."

Características de la red 12 social disciplinar

Momento de uso de la red social disciplinar
“Esa finalidad declarada de que sea con fines académicos ayudaría de entrada a filtrar quien va a ser parte de esa red social creo ahí ya decanta todo"

"Que esta red les pueda permitir no sé si pudiera lograr que solo tenga acceso a la información sino incluso que puedan hacer ciertos modelamientos, a lo mejor en una actividad que el alumno pueda hacer un pequeño video o audio de como planear su negociación, así dentro de los lineamientos que ustedes están, como que los llevan a un modelaje. Por ejemplo como iniciar la entrevista."
"Se vuelve también un espacio en donde me parece que se va más a la queja como más al desahogo pero no al aplicar alguna solución."
Uso poco ético de otras 9
redes

8

Responsabilidad en el uso de redes sociales

Reglas y normas de las redes sociales disciplinares

Lugar de práctica

Desventajas de las redes sociales comunes

Ventajas de las redes sociales comunes
“Al final es parte de su ética como parte de su formación ética, entonces me imagino que así como cuando pedimos también que en los foros sean respetuosos con el lenguaje, etc., pues ustedes también tienen lineamientos muy concretos y que se basen en la ética."

“Sí que los estudiantes hagan uso responsable de la información que les van a proporcionar y también de lo que ellos van a compartir, que realmente se vea el compromiso y la seriedad para su módulo y que sus participaciones tengan esa intención de su proceso de aprendizaje y no como una cuestión vana."

“Les pedimos que vayan a un centro que de forma como eje de sus servicios ofrezcan educación especial."

"Porque muchas veces las páginas de Facebook es al menos por lo que he visto es alguien que las crea, las avienta a Facebook y ya se desatiende y se vuelve una cosa que no tiene ni pies ni cabeza, entonces pienso que alguien tiene que administrar e ir controlando la situación."

“Las herramientas que ya existen de redes sociales Facebook, Twitter, Instagram creo que son bastantes útiles el simple hecho de poder compartir archivos, fotografías, videos, materiales digitales eso ya es útil para fines académicos que puedan agregarse otras herramientas como los cuestionarios, las encuestas eso también esta padrísimo para fines académicos." mismos docentes, además deben ser leídos y aceptados por toda la comunidad de participantes.

Comunidad: Estudiantes y profesores que conforman la red, los cuales estarían en constante interacción dentro de la red.

División del trabajo: Son los roles que los participantes pueden tomar, en este caso los profesores como moderadores de la red, gestionando la actividad e interacción, al mismo tiempo que fungen como expertos y asesores de las prácticas de los estudiantes. Los estudiantes fungen como participantes y brindan apoyo entre pares.

Este análisis desde la Teoría de la Actividad permite visualizar la interrelación entre los diferentes actores y elementos, todos enfocados en alcanzar el objetivo común que es la construcción de conocimiento en los módulos propedéuticos.

Sin embargo, el principal resultado obtenido de las entrevistas son las tensiones en el sistema, que en este caso en particular puede ser la duración de la participación de los estudiantes en la red, no habiendo consenso entre los docentes, dado que un docente afirma que sería deseable que estuviera abierta todo el semestre, mientras que los demás docentes sugiere que sea sólo para un uso específico, acotado en el tiempo (al inicio del semestre). Esto puede generar, una vez realizada la implementación, en una discordancia entre la propuesta 


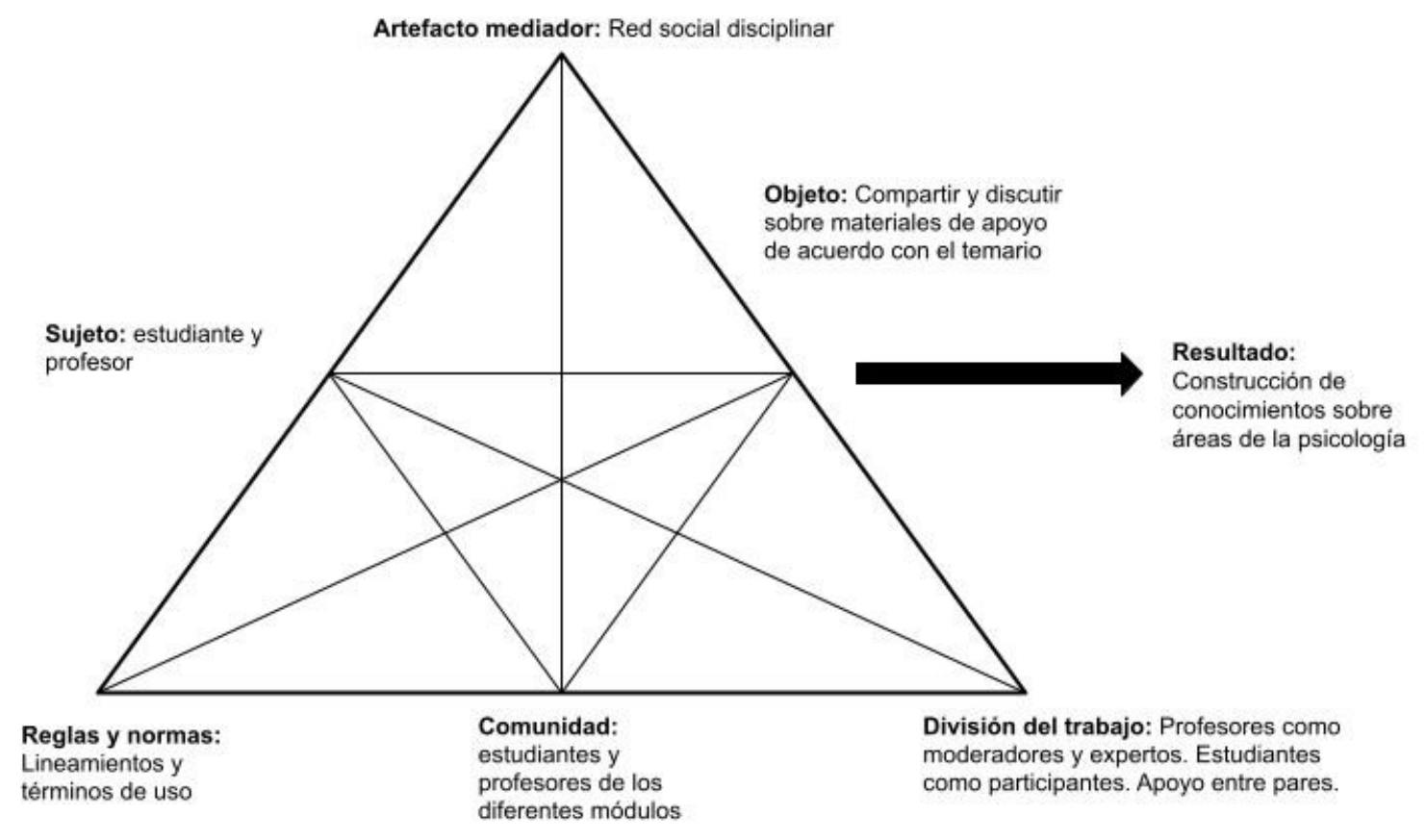

Figura 2

Elementos de la Teoría de la actividad en la red social disciplinar. Elaboración propia

de uso y verse reflejada en la discrepancia en el objeto de uso: compartir y discutir sobre materiales de apoyo en asignaturas prácticas de los módulos propedéuticos.

\section{Discusión}

El objetivo de esta investigación fue analizar las posibilidades de uso de una red social disciplinar desde la perspectiva de los docentes. Como pudo revisarse, los cuatro docentes entrevistados consideraron como algo favorable la participación de los alumnos en este tipo de redes, creando una comunidad en este tipo de redes, específicamente para compartir información e inclusive dar soporte emocional, teniendo claridad en el objetivo de uso y en las normas y reglas de la red. También se encontraron diversas dificultades para la práctica que a su vez pueden verse hasta cierto punto apoyadas para un buen desarrollo empleando una red social disciplinar, como lo es el compartir experiencias, tener un espacio de modelamiento por parte de los docentes y un espacio para compartir información disciplinar y académica en un lugar seguro, en donde inclusive se aborde como un espacio de formación ética para el uso responsable de redes sociales por parte de la comunidad de estudiantes y profesores.

Estas respuestas se alinean con las experiencias reportadas por Cunha Jr. et al. (2016) quienes emplearon la Teoría de la Actividad para realizar un análisis sobre el uso de Facebook en el ámbito educativo, concluyendo que es importante enfatizaron en el desarrollo y establecimiento de roles, normas y reglas explícitas claras para todos los participantes de una red social empleada con un énfasis educativo, pues mencionan que estas normas deben ser empleadas para el desarrollo de las actividades. En cuanto a los roles, sugieren que las figuras docentes sean quienes establezcan estas reglas y normas, fungiendo también como moderadores.

Por otro lado, autores como Bagarukayo, Sentamu, Mayisela y Brown (2016), afirman que a partir del uso de redes sociales se puede promover el aprendizaje al compartir información e interactuar entre profesores y estudiantes, sin embargo, también señalan que pueden aparecer tensiones, desde el punto de vista de la CHAT, como la falta de acceso a las herramientas pertinentes (teléfono o computadora, acceso a internet) por parte de algunos miembros de la comunidad, por lo que su resolución cambia el sistema general. Lo anterior reafirma la intención de emplear la CHAT como un marco teórico-metodológico para el análisis de la práctica educativa mediada por tecnología, pues a futuro permitiría dar cuenta de estas tensiones y de los cambios históricos a lo interno del sistema, en este caso, si se llegara a la implementación de la red social.

Respecto al tipo de red social a emplear, la mayoría de las experiencias reportadas en la literatura emplean 
la red social como Facebook (Oliva, 2017), de hecho, esa misma red social es la que emplean la mayoría de los estudiantes de la licenciatura en línea SUAyED, sobre todo para mantener vínculos fuera de las aulas virtuales entre compañeros, mientras que también se encontró una favorable intención de los estudiantes para participar en una red social disciplinar (Meza, Miranda y Delgado, 2015). Sin embargo, ya se han señalado algunas de las principales problemáticas inherentes a ese tipo de redes privativas, como son las precepciones de riesgo respecto a seguridad y privacidad (Marín y Cabero, 2019), o también sobre su estructura centralizada por empresas que alojan y controlan la información, dejando de lado el objetivo inicial con el que fueron creadas, es decir, generar redes sociales centradas en las personas, para hacer énfasis en redes sociales centradas en las empresas (La Cava, Greco y Tagarelli, 2021).

Es por esta razón que se considera importante que las universidades promuevan el uso de las Redes Sociales de código abierto para crear así comunidades disciplinares en las que se pueda discutir sobre temas académicos, permitiendo el desarrollo de conocimientos en interacción. En este estudio, los profesores entrevistados, están de acuerdo con que se trate de una red social de código abierto y moderada por los mismos miembros de la comunidad de profesores, lo que permitiría lo mencionado por Cabrera (2017), sobre las redes sociales libres en las universidades, puesto que permitirían la gestión de contenidos, la administración de usuarios y el desarrollo e implementación de lineamientos de uso.

Esto viene a colación a partir de situaciones en donde las redes sociales privativas más empleadas han sido señaladas por diversas instituciones como espacios poco seguros, como lo que sucedió con WhatsApp el 4 de enero de 2021, a partir de un anuncio del Instituto Nacional de Transparencia, Acceso a la Información y Protección de Datos Personales (INAI, 2021) de México, quien recomendó ampliamente a los usuarios leer las políticas de privacidad y tomar decisiones sobre su uso dado que parecían poco transparentes e invasivas para la privacidad. Esto es importante dado que en el área educativa las redes sociales más frecuentemente empleadas son Facebook y Whatsapp (Cetinkaya y Sütçü, 2018).

\section{Conclusiones}

Como conclusión es importante señalar que los módulos propedéuticos tienen un gran peso en la formación de los futuros psicólogos, puesto que en ellos se reflejan los conocimientos adquiridos en semestres anteriores y se realiza la primera práctica como acercamiento a cada uno de los programas de profundización que brinda la licenciatura (Universidad Nacional Autónoma de México, 2005), por lo que sería deseable que fuera una experiencia favorable y satisfactoria. Según los hallazgos de este estudio, para la propuesta de uso de la red social disciplinar, los docentes afirman que es conveniente mantenerla activa y abierta durante la totalidad del semestre con el objetivo de que los estudiantes tengan una vía de apoyo, tanto de profesor-alumno, como de apoyo entre pares, generando así una red de soporte, enfatizando siempre las posibilidades y limitantes señalados en los términos de uso de la red, los cuales deberán ser claros y difundidos a todos los miembros de la comunidad de usuarios.

A futuro, sería deseable ampliar el uso de la red social disciplinar, por ejemplo para su uso en aquellos módulos identificados con mayor índice de reprobación o bien para los módulos de práctica en los semestres cercanos al egreso, con el objetivo de que los estudiantes puedan compartir su experiencia o compartir dificultades durante sus intervenciones o investigaciones.

Por último, entre las problemáticas presentadas en este estudio destaca la cantidad de entrevistas, pues el contar con cuatro profesores deja fuera otras perspectivas del fenómeno, por lo que es pertinente, en el futuro, entrevistar a una muestra mayor de docentes, no sólo de los módulos propedéuticos sino también de otros módulos y áreas de la carrera de psicología. También es conveniente analizar si el uso de una red social disciplinar puede tener un impacto en las actividades académicas colaborativas, en contraste con otras herramientas como lo serían los foros de discusión en la plataforma Moodle, dado que es el principal medio de interacción en la educación formal a distancia. Esto podría abrir una nueva forma de interacción y comunicación en beneficio del aprendizaje mediado por tecnología

\section{Referencias}

Bagarukayo, E., Ssentamu, P., Mayisela, T., \& Brown, C. (2016). Activity Theory as a lens to understand how Facebook develops knowledge application skills. International Journal of Education and Development using Information and Communication Technology, 12(3), 128-140. https:// files.eric.ed.gov/fulltext/EJ1124826.pdf

Cabrera, E. (2017). Redes sociales libres en la universidad pública. Revista digital universitaria. 18(1).http:// paginaspersonales.unam.mx/app/webroot/files/5379/ Publica_20180215212249.pdf

Cetinkaya, L., \& Sütçü, S. S. (2018). The effects of Facebook and WhatsApp on success in English vocabulary instruction. Journal of Computer Assisted Learning, 34(5), 504-514. https://doi.org/10.1111/jcal.12255 
Cunha Jr., F., Van Oers, B. \& Kontopodis, M. (2016). Collaborating on facebook: Teachers exchanging experiences through social networking sites. Cultural-Historical Psychology, 12(3), 290-309.https://doi.org/10.17759/ chp. 2016120318

Engeström, Y.(2001). El aprendizaje expansivo en el trabajo: hacia una reconceptualización teoría de la actividad. Journal of Education and Work, 14(1). https://www.bibliopsi. org/docs/carreras/obligatorias/CFG/05general/ adesignar/Engestrom\%20-\%20El\%20Aprendizaje\%20 Expansivo\%20en\%20El\%20Trabajo.pdf

Engeström, Y. (2009). Expansive Learning. Toward an activitytheoretical reconceptualization. En: Illeris, K. (Ed). Contemporary Theories of Learning. Nueva York: Estados Unidos: Routledge.

Erausquin, C. (2014). La Teoría histórico-cultural de la Actividad como artefacto mediador para construir intervenciones e indagaciones sobre el trabajo de psicólogos en escenarios educativos. Revista Segunda Época, 13. 173-197.https:// www.aacademica.org/cristina.erausquin/374.pdf

García, R. (2017). Aplicabilidad de la Teoría de la Actividad Histórico-Cultural en los estudios de comportamiento informacional. Biblios, (67), 69-83. http://dx.doi. org/10.5195/biblios.2017.336

INAI (2021). INAI Ilama a usuarios de whatsapp a revisar a detalle nuevos términos de política de privacidad y tratamiento de datos personales. https://home.inai.org.mx/wpcontent/documentos/SalaDePrensa/Comunicados/ Comunicado\%20INAI-014-21.pdf

La Cava, L., Greco, S., \& Tagarelli, A. (2021). Understanding the growth of the Fediverse through the lens of Mastodon. Applied Network Science, 6(1), 1-35. https://doi. org/10.1007/s41109-021-00392-5

Kirschner, P. A. (2015). Facebook as learning platform: Argumentation superhighway or dead-end street?. Computers in Human Behavior, 53, 621-625. https://doi. org/10.1016/j.chb.2015.03.011

Larripa, M. \& Erausquin, C. (2008). Teoría de la actividad y modelos mentales. Instrumento para la reflexión sobre la práctica profesional: "Aprendizaje expansivo", intercambio cognitivo y transformación de intervenciones de psicólogos y otros agentes en escenarios educativos. Anuario de investigaciones, XV. https://www.redalyc. org/pdf/3691/369139944009.pdf

Marín, V. \& Cabero, J. (2019) Las redes sociales en educación: desde la innovación a la investigación educativa. RIED. Revista Iberoamericana de Educación a Distancia, 22 (2), 25-33. https://doi.org/10.5944/ried.22.2.24248

Meza, J., Miranda, G., \& Delgado, Z. (2015) Resultados exploratorios sobre las características posibles de una red social académica desde la perspectiva de los estudiantes.
En XIII Congreso nacional de investigación educativa 2015, Chihuahua. http://www.comie.org.mx/congreso/ memoriaelectronica/v13/doc/1155.pdf

Meza, J., Miranda, G., \& Delgado, Z. (2019) Percepción de uso de redes sociales disciplinares por parte de estudiantes. En XV Congreso nacional de investigación educativa COMIE-2019. San Luis Potosí. http://www.comie.org.mx/ congreso/memoriaelectronica/v15/doc/2790.pdf

Miranda, A. (2015) EDUSOL. Tensiones y síntesis de una comunidad virtual de aprendizaje. México: Educación, cultura y software libre. https://chat.iztacala.unam.mx/ index.php/libro/edusol-tensiones

Muñoz, M. M. (2018). Virtualización del espacio público y concepto débil de privacidad. Lecciones del caso Facebook-Cambridge Analytica. Ensayos de Filosofía, 8(2). https://www.ensayos-filosofia.es/archivos/articulo/ virtualizacion-del-espacio-publico-y-concepto-debilde-privacidad-lecciones-del-caso-facebook-cambridgeanalytica

Oliva, H. (2017). Redes sociales como componentes educativos en los entornos digitales de aprendizaje. Realidad y reflexión, 46. https://doi.org/10.5377/ryr.v0i46.5506

Ormart, E., \& Navés, F. (2014). El uso de las redes sociales como soporte educativo. CPU-e Revista de investigación educativa, $\quad 18$. https://doi.org/10.25009/cpue. v0i18.760

Peña, K., Pérez, M. \& Rondón, E. (2010). Redes sociales en Internet: Reflexiones sobre sus posibilidades para el aprendizaje cooperativo y colaborativo. Revista de teoría y didáctica de las ciencias sociales, (16), 173-205.https:// www.redalyc.org/pdf/652/65219151010.pdf

Salas, F. (2016). Aportes del modelo de Yrjö Engeström al desarrollo teórico de la docencia universitaria. Revista Educación, 40(2),1-22. https://doi.org/10.15517/ revedu.v40i2.15257

Suárez, K. V. M., Carreño, E. J. M., \& Rivero, A. J. M. (2017). Las redes sociales y su importancia en la Educación Superior. Opuntia Brava, 9(4), 284-298. http://200.14.53.83/ index.php/opuntiabrava/article/view/230

Universidad Nacional Autónoma de México (2005) Proyecto de modificación al plan de estudios vigente de la Licenciatura en Psicología del Sistema Escolarizado para impartirse en Sistema Universidad Abierta y Educación a Distancia en la FES-Iztacala. Capítulo 7 https://psicologia.iztacala. unam.mx/cambio_curricular/periodosilva/documentos/ proyecto_modificacion.htm\#ocho

Vázquez, A. \& Cabero, J. (2015). Las redes sociales aplicadas a la formación. Revista Complutense de Educación, 26, 253-272. http://dx.doi.org/10.5209/rev_RCED.2015. v26.47078 


\section{Meta-Análisis del Artículo}




\section{Dimensión Cuantitativa}

\section{Perfil de Evaluación entre pares}
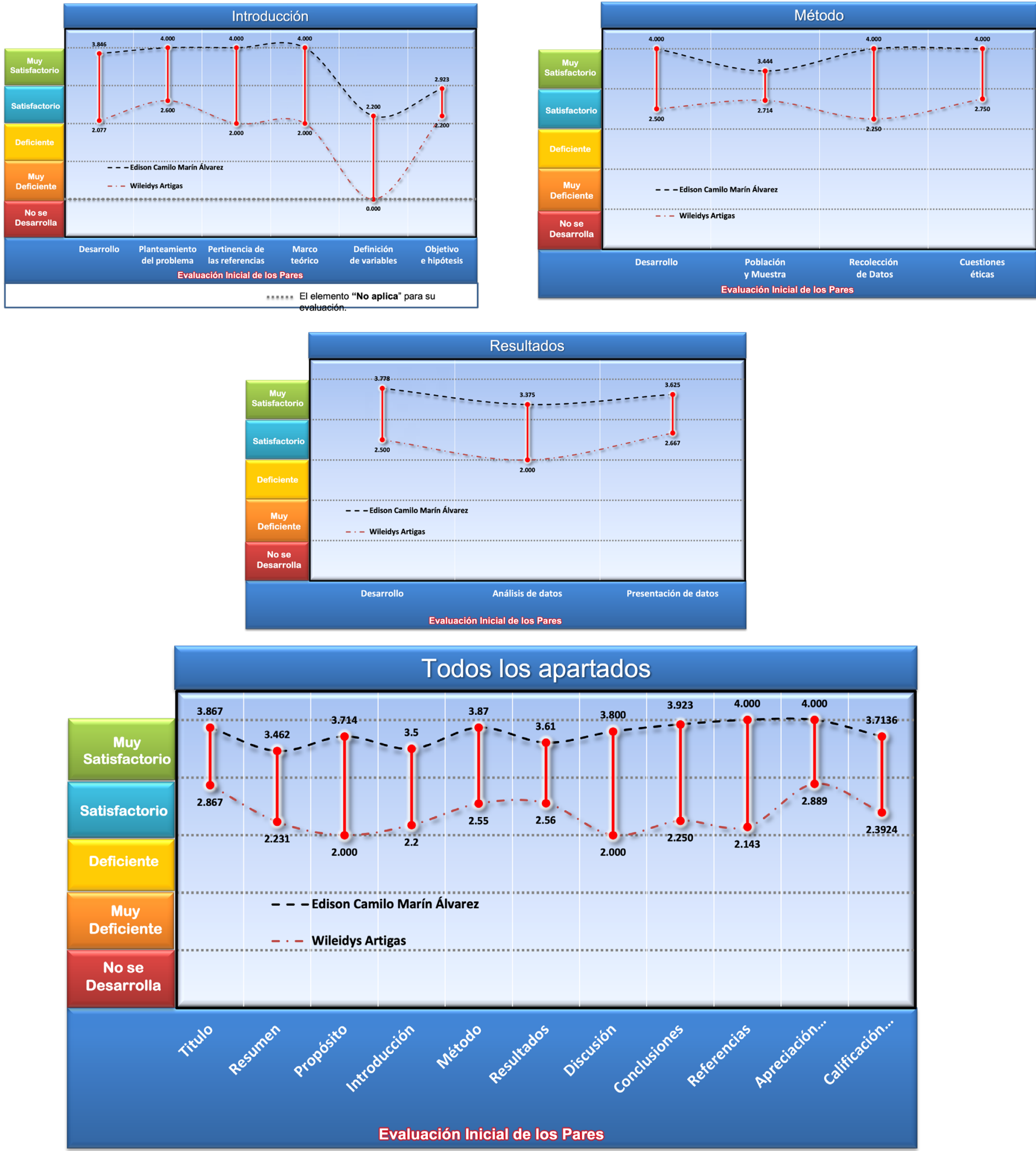


\section{Índice de Concordancia}

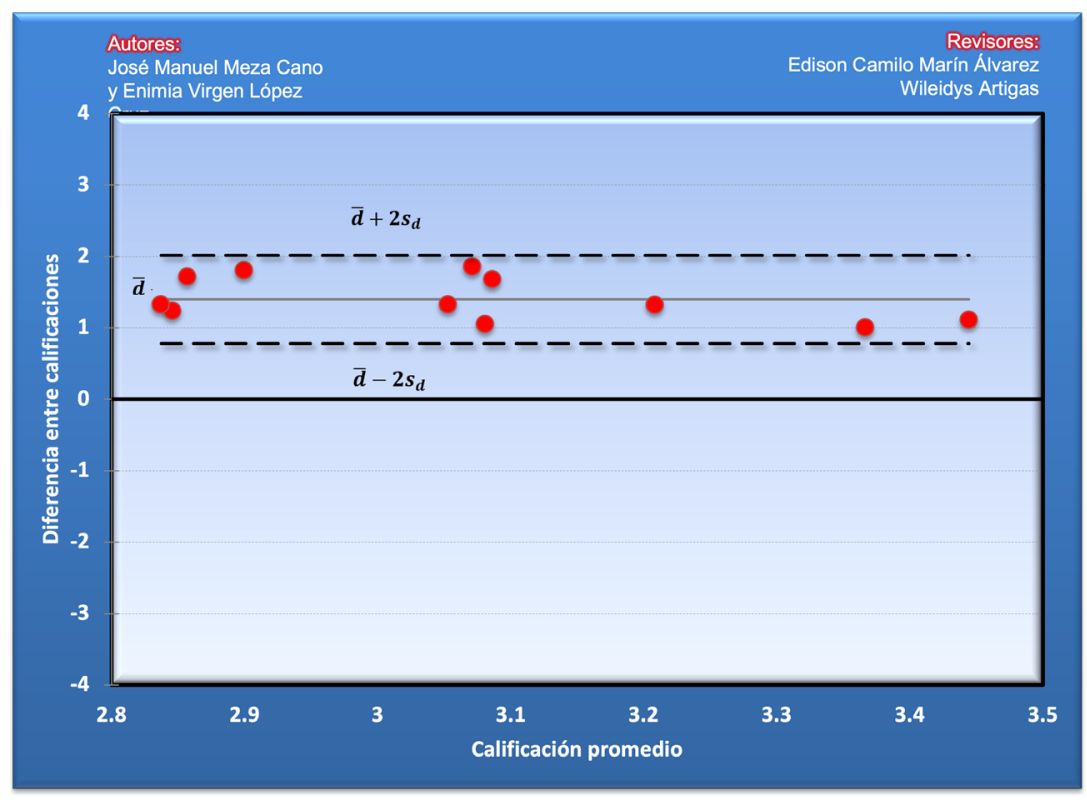

Índice de Acuerdo

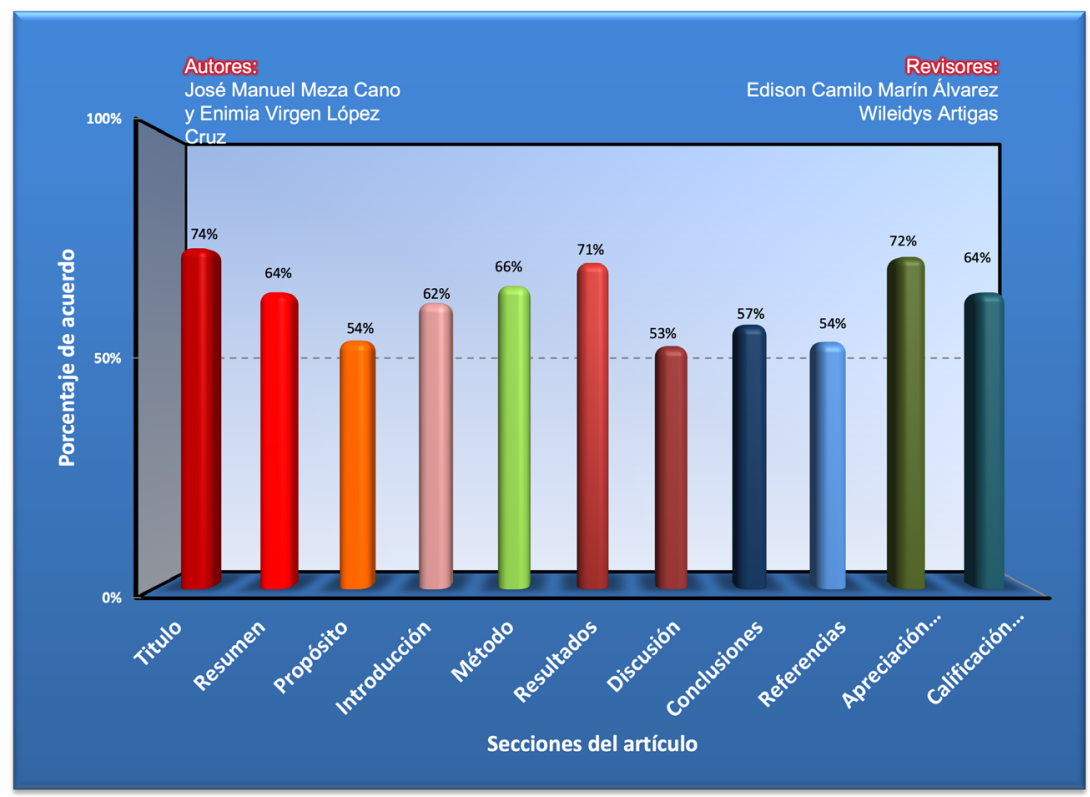




\begin{tabular}{|c|c|}
\hline Revisor 1 & Revisor 2 \\
\hline Edison Camilo Marín Alvarez & Wileidys Artigas \\
\hline \multicolumn{2}{|c|}{ Título/Autoría } \\
\hline $\begin{array}{l}\text { Se podría enriquecer el titulo para que hiciera referencia } \\
\text { o tuviera un poco de acercamiento al objetivo propuesto. }\end{array}$ & $\begin{array}{l}\text { El titulo debe dejar claro que no hay una aplicación del } \\
\text { proceso, yo sugeriria quitarle la palabra propuesta }\end{array}$ \\
\hline \multicolumn{2}{|c|}{ Resumen } \\
\hline $\begin{array}{l}\text { El artículo no presenta valores numericos pero se en- } \\
\text { tiende que se trata de un estudio cualitativo de orden } \\
\text { exploratorio. }\end{array}$ & $\begin{array}{l}\text { Aunque el resumen es bastante concreto, las normas seña- } \\
\text { las } 200 \text { palabras y este formulario habla de 150, por otro } \\
\text { lado la última palabra clave hay que revisarla }\end{array}$ \\
\hline \multicolumn{2}{|c|}{ Próposito del Estudio } \\
\hline $\begin{array}{l}\text { Se podría hacer un poco más explicito el proposito del } \\
\text { estudio fortaleciendo el objetivo propuesto. }\end{array}$ & $\begin{array}{l}\text { Creo que el proposito debe ser aclarado en función del } \\
\text { contenido }\end{array}$ \\
\hline \multicolumn{2}{|c|}{ Introducción } \\
\hline $\begin{array}{l}\text { Se podrían expresar de manera más explicita las variables } \\
\text { e hipotesis. }\end{array}$ & $\begin{array}{l}\text { Es importante señalar que siendo un tema de tanta } \\
\text { actualidad existen estudios relacionados que pueden } \\
\text { ayudar a enriquecer la parte teorica, empezando por } \\
\text { la relación con las Redes Sociales Academicas existen- } \\
\text { tes. Las fuentes que se utilizan son pocas, existen re- } \\
\text { ferencias actuales importantes en el tema que podrían } \\
\text { orientar el desarrollo. No se muestran antecedentes al } \\
\text { respecto de esta propuesta. }\end{array}$ \\
\hline \multicolumn{2}{|c|}{ Método } \\
\hline $\begin{array}{l}\text { Sería positivo describir la cantidad total de profesores } \\
\text { hacer parte de los cursos de práctica. }\end{array}$ & $\begin{array}{l}\text { La parte metodologica requiere revisión, iniciando por el } \\
\text { bajo tamaño de la muestra. El instrumento no presenta un } \\
\text { basamento teorico de donde fue obtenido, parece pregun- } \\
\text { tas lanzadas al respecto del tema }\end{array}$ \\
\hline
\end{tabular}




\begin{tabular}{|c|c|}
\hline Revisor 1 & Revisor 2 \\
\hline \multicolumn{2}{|c|}{ Resultados } \\
\hline $\begin{array}{l}\text { Es claro que el articulo es de corte cualitativo pero algunos } \\
\text { datos estadisticos podrían fortalecerlo. }\end{array}$ & $\begin{array}{l}\text { Los resultados presentan una de las deficiencias del texto, } \\
\text { no hay mayor analisis o ampliación y contrastación que } \\
\text { permita observar el estudio como relevante e importante } \\
\text { en su área de estudio. }\end{array}$ \\
\hline \multicolumn{2}{|c|}{ Discusión } \\
\hline Algunas hipotesis podrían enriquecer el escrito. & La discusión no esta ordenada, ni es suficiente \\
\hline \multicolumn{2}{|c|}{ Conclusiones } \\
\hline $\begin{array}{l}\text { El estudio señala claramente la necesidad de incluir más } \\
\text { docentes en el proceso de recolección de información. }\end{array}$ & $\begin{array}{l}\text { Las conclusiones podrían mejorar si el estudio comple- } \\
\text { to es ampliaro }\end{array}$ \\
\hline \multicolumn{2}{|c|}{ Referencias } \\
\hline Las citas y referencias son adecuadas. & Revisar comentarios de texto \\
\hline
\end{tabular}




\section{Historia del Proceso}

\section{EDITORIAL}

